\title{
The ground state of the Kondo model with large spin
}

\author{
Shun-Qing Shen \\ Max Planck Institute for Physics of Complex Systems, Bayreuther Strasse 40, Haus 16, D- 01187 Dresden, Germany
}

(Received: December 26, 1996)

\begin{abstract}
In this paper, we prove that the ground state of the Kondo model with large spin is nondegenerate, apart from a $\mathrm{SU}(2)$ spin degeneracy in the case of half filling. The ground state spin is found for the system, and the energy level orderings are discussed. Finally, the existence of ferrimagnetism in some cases is proved.
\end{abstract}

PACS numbers: 75.10.Lp 75.30.Mb, 75.50.Gg

The Kondo models, or single and lattice impurity models, are one of the most challenging subjects in strongly correlated systems 13 . Recent developments of reflection positivity technique in the spin space make it possible to establish some rigorous results for the half-filled strongly correlated electron systems 4 . Theory of reflection positivity in the spin space for the single- and multi-channel Kondo models with spin $1 / 2$ was developed recently and a series of rigorous results on the ground state properties were proved 89. However some materials are described by the Kondo models with large spin, such as $\left(\mathrm{La}_{1-x} X_{x}\right) \mathrm{MnO}_{3}$ with $\mathrm{X}=\mathrm{Ba}, \mathrm{Ca}, \mathrm{Sr}$ etc. and the localised spin $s=3 / 210$. Due to more degrees of freedom in the case of large spin than in the case of spin $1 / 2$, usually it is very hard to extract rigorous results for those systems. As a generalisation of the theory for the Kondo model with spin $1 / 2$, we will investigate the Kondo model with large spin and provide some rigorous results on the ground state of the Kondo model in the case of half filling.

Let us first write down the Hamiltonian we will investigate:

$$
\begin{aligned}
H & =\sum_{\langle i j\rangle \in \wedge, \sigma} t_{i j} c_{i, \sigma}^{\dagger} c_{j, \sigma}+\sum_{i \in \wedge} U_{i}\left(n_{i, \uparrow}-\frac{1}{2}\right)\left(n_{i, \downarrow}-\frac{1}{2}\right) \\
& +\sum_{i \in \wedge} J_{i} \mathbf{S}_{i} \cdot \mathbf{S}_{c i}+\sum_{i j \in \wedge_{d}} K_{i j} \mathbf{S}_{i} \cdot \mathbf{S}_{j}
\end{aligned}
$$

where $c_{i, \sigma}^{\dagger}$ and $c_{i, \sigma}$ are the creation and annihilation operators for the conduction electron (c-electron) at site $i$ with spin $\sigma(=\uparrow, \downarrow)$ and $n_{i, \sigma}=c_{i, \sigma}^{\dagger} c_{i, \sigma} . \quad \mathbf{S}_{i}$ is the localised spin operator with $\operatorname{spin} s_{i}$ at site $i$. $\mathbf{S}_{c i}=$ $\sum_{\sigma, \sigma^{\prime}} c_{i, \sigma}^{\dagger} \boldsymbol{\sigma}_{\sigma, \sigma^{\prime}} c_{i, \sigma^{\prime}} / 2$, and $\boldsymbol{\sigma}_{\alpha}(\alpha=x, y, z)$ are the Pauli matrices. The model is defined on a bipartite lattice $\wedge$ with the site numbers $N_{A}$ and $N_{B}$ of the two sublattices $A$ and $B . t_{i j}=t_{j i}$ is possibly non-zero only when $i$ and $j$ belong to two different sublattices. The lattice $\wedge$ is connected by the hopping terms $\left\{t_{i j}\right\}$, which implies that for any two sites $k$ and $l$ on $\wedge$, we can always find a sequence $\left\{\left(k, i_{1}\right),\left(i_{1}, i_{2}\right), \cdots,\left(i_{n}, l\right)\right\}$ such that $t_{k i_{1}} t_{i_{1} i_{2}} \cdots t_{i_{n} l} \neq 0$. $\wedge_{d}$ is the distribution of localised spins on $\wedge$. If we regard the same site for conduction electron and localised spin as two independent sites in a generalised lattice, the site $i$ for conduction electron and the site $i$ for localised spin belong to the same sublattice when $J_{i}<0$, and to two different sublattice when $J_{i}>0$. In the case the generalised lattice can be still regarded as a generalised bipartite lattice. Assume $K_{i j} J_{i} J_{j} \leq 0$ if $i$ and $j$ belong to the same sublattice, and $K_{i j} J_{i} J_{j} \geq 0$ if $i$ and $j$ belong to two different sublattices. When $J_{i}$ and $J_{j}$ are constant or have the same sign, $K_{i j} \leq 0$ if $i$ and $j$ belong to the same sublattice and $K_{i j} \geq 0$ if $i$ and $j$ belong to two different sublattices as in the antiferromagnetic Heisenberg model on a bipartite lattice. This condition guarantees that the last term in Eq.(1) does not introduce any frustration for the generalised bipartite lattice. Physically, it is completely absent of frustration in this case. The model is reduced to the Hubbard model when $\wedge_{d}=0$, i.e., there is no magnetic impurity or localised spin in the systems. The structure function of the bipartite lattice $\wedge$ is defined as $\epsilon(i)=1$ if $i \in A$ and -1 if $i \in B$.

The main results are summarised as follows:

Theorem: Assume the model in Eq.(1) with $t_{i j}$ and $K_{i j}$ is defined on a connected bipartite lattice $\wedge$ with sublattice sites $N_{A}$ and $N_{B}$ and with the distribution of localised spins $\wedge_{d}$. All $U_{i}>0$ and $J_{i} \neq 0$ and the number of conduction electrons is $N_{e}=N_{\wedge}=N_{A}+N_{B}$. The signs of $K_{i j}$ and $J_{i}$ satisfy the previously stated condition. Denote the lowest energy state in the subspace decomposed by the z-component of the total spin $S_{t o t}^{z}$ by $|\Psi\rangle$.

i). The state $|\Psi\rangle$ is non-degenerate in each subspace of $S_{\text {tot }}^{z}$. The ground state of Eq. (1) is unique apart from a $\left(2 S_{t o t}^{0}+1\right)$-fold spin degeneracy.

ii). The total spin $S_{t o t}$ in the lowest energy state is

$$
S_{t o t}= \begin{cases}S_{t o t}^{0}, & \text { if }\left|S_{t o t}^{z}\right| \leq S_{\text {tot }}^{0} \\ \left|S_{\text {tot }}^{z}\right|, & \text { otherwise }\end{cases}
$$

where

$$
S_{\text {tot }}^{0}=\left|\frac{1}{2} \sum_{i \in \wedge} \epsilon(i)-\sum_{i \in \wedge_{d}} s_{i} \frac{J_{i}}{\left|J_{i}\right|} \epsilon(i)\right| .
$$

iii). The spin-spin correlation functions obey,

$$
\begin{aligned}
& \left\langle\Psi\left|\mathbf{S}_{c i}^{+} \cdot \mathbf{S}_{c j}^{-}\right| \Psi\right\rangle=\epsilon(i) \epsilon(j) C_{i j} \\
& \left\langle\Psi\left|\mathbf{S}_{i}^{+} \cdot \mathbf{S}_{j}^{-}\right| \Psi\right\rangle=\epsilon(i) \epsilon(j) F_{i j} \\
& \left\langle\Psi\left|\mathbf{S}_{c i}^{+} \cdot \mathbf{S}_{j}^{-}\right| \Psi\right\rangle=-\epsilon(i) \epsilon(j) \frac{J_{\perp}}{\left|J_{\perp}\right|} G_{i j},
\end{aligned}
$$


where $C_{i j}, F_{i j}$ and $G_{i j} \geq 0$ if $U_{i} \geq 0$, and $>0$ if all $U_{i}>0$.

iv). When all $U_{i}=0$, (at least one of) the ground state(s) (if degenerate) has the total spin as that in Eq. (2).

Before we present the proof, several remarks or corollaries are made:

$1)$. In the case of all $J_{i}>0$ or $<0$ and $s_{i}=s$, suppose that $N_{A d}$ spins are on the sublattice $A$, and $N_{B d}$ spins on the sublattice $B$, the total spin is

$$
S_{\text {tot }}^{0}=\left|\frac{1}{2}\left(N_{A}-N_{B}\right)-\frac{J}{|J|} s\left(N_{A d}-N_{B d}\right)\right| .
$$

In the Kondo lattice case, $N_{A}=N_{A d}$ and $N_{B}=N_{B d}$, and the total spin is

$$
S_{t o t}^{0}=\left(s-\frac{1}{2} \frac{J}{|J|}\right)\left|N_{A}-N_{B}\right| .
$$

When all $s_{\text {ir }}=1 / 2$, we recover the result for the case of spin $1 / 2$ 目 9 . The ground state is a singlet only when $N_{A}=N_{B}$, or $s=1 / 2$ and $J>0$. When $N_{A} \neq N_{B}$ and $\left(N_{A}-N_{B}\right) /\left(N_{A}+N_{B}\right) \neq 0$ when the system becomes sufficiently large, we obtain a state with ferromagnetic long-range order.

2). Theorem (iii) indicates that strong antiferromagnetic correlations exist between the conduction electrons or localised spins. A direct corollary is that the antiferromagnetic correlation is always stronger than the ferromagnetic correlation. For example on a cubic lattice,

$$
\left\langle\mathbf{S}_{Q}^{+} \cdot \mathbf{S}_{Q}^{-}\right\rangle=\max \left\{\left\langle\mathbf{S}_{q}^{+} \cdot \mathbf{S}_{-q}^{-}\right\rangle\right\},
$$

where $\mathbf{S}^{+}=\frac{1}{\sqrt{N_{\curlywedge}}} \sum_{i \in \Lambda} \mathbf{S}_{i}^{+} e^{i \vec{q} \cdot \overrightarrow{r_{i}}}$ and $Q=(\pi, \pi, \cdots)$. If the state possesses ferromagnetic long-range order, it must also possess antiferromagnetic long-range order. In other words, the ferromagnetism in the case of $N_{A} \neq N_{B}$ is, strictly speaking, the ferrimagnetism.

$3)$. The energy level orderings can be obtained from Theorems (i-ii) and the $\mathrm{SU}(2)$ symmetry with a variational principle: denote $E\left(S_{\text {tot }}^{z}\right)$ the lowest energy state with $S_{\text {tot }}^{z}$. As $\left|\Psi\left(S_{t o t}^{z}\right)\right\rangle$ is the lowest energy state with $S_{t o t}^{z}$, we can construct an eigenstate $\left(\sum_{i \in \Lambda} \mathbf{S}_{c i}^{-}+\right.$ $\left.\sum_{i \in \wedge_{d}} \mathbf{S}_{i}^{-}\right)\left|\Psi\left(S_{\text {tot }}^{z}\right)\right\rangle$ (suppose $S_{\text {tot }}^{z} \geq 1$ ) due to the spin $\mathrm{SU}(2)$ symmetry. This state has its z-component of the total spin $S_{\text {tot }}^{z}-1$ and the eigenvalue $E\left(S_{t o t}^{z}\right)$. Meanwhile it has the same total spin as in $\left|\Psi\left(S_{\text {tot }}^{z}\right)\right\rangle$. In the variational principle the lowest energy state in the subspace of $S_{t o t}^{z}-1$ should not be higher than $E\left(S_{t o t}^{z}\right)$. If the lowest energy in the subspace $S_{t o t}^{z}-1$ is equal to the lowest energy in the subspace $S_{t o t}^{z}$, the lowest energy state or one of the states if degenerate with $S_{t o t}^{z}-1$ must has the same total spin in the state with $S_{t o t}^{z}$. Since the lowest energy states in each subspace are non-degenerate when all $U_{i}>0$ and we assume $S_{\text {tot }}^{z} \geq 0$, we have

$$
\begin{aligned}
& E\left(S_{t o t}^{z}\right)=E\left(-S_{t o t}^{z}\right) ; \\
& E\left(S_{t o t}^{z}\right)<E\left(S_{t o t}^{z}+1\right) \text { if } S_{t o t}^{z} \geq S_{t o t}^{0} \\
& E\left(S_{t o t}^{z}\right)=E\left(S_{t o t}^{0}\right) \quad \text { if } 0 \leq S_{t o t}^{z} \leq S_{t o t}^{0} .
\end{aligned}
$$

For $S_{t o t}^{z} \leq 0$, the energy level ordering can be obtained from Eqs. (8-10). This is similar to that of the Heisenberg modeli2 2 . It is worth of mentioning that in the case of $U_{i} \geq 0$ we have to use "lower or equal" instead of "lower than" if we could not determine whether the lowest energy state in each subspace is non-degenerate or not

4). In the one-dimensional chain of the Kondo lattice with all $J_{i}>0$ or $<0$, Theorems (ii) and (iii) on the total spin and spin-spin correlation functions are the same as those in a two-chain spin-ladder system. This coincides with the analysis on the resemblance of these two systems by White and Affleck 13 .

$5)$. When the system has no localised spin or magnetic impurity, $N_{A d}=N_{B d}=0$ and the model in Eq.(1) is reduced to a Hubbard model. Theorem (i-iv) hold for the Hubbard model. In the case, one can regard the spin formula in Eq. (2) as ageneralisation of Lieb's theorem for the Hubbard modelt. The conditions of even number of the lattice sites and $S_{t o t}^{z}=0$ in Lieb's theorem are removed. As a corollary, the energy level ordering for the Hubbard model in the case of half filling is the same as shown in Eqs. (8-10).

The main steps to prove this theorem are 1). to express the spin operator in a multi-fermion representation; 2 ). to define the generalised bipartite lattice after we consider the localised spins; 3 ) to introduce a complete and orthonormal set of basis for the system; 4) to prove the positive definiteness or semidefiniteness of the ground state on the chosen basid 14 ; and 5) to prove (iii) and (iv) by utilising the positive definiteness of the ground state and the known theorems on a positive definite state.

Except for the reflection positivity approach in the spin space as in the case of spin $1 / 2$. 0 , the key trick used in this paper is to express the localised spin operator $\mathbf{S}_{i}$ as the summation of $2 s_{i}$ spin $1 / 2$ operators in a multi-fermion representation (d-fermion) and these $2 s_{i}$ spins are coupled ferromagnetically. Completely ferromagnetic coupling could be realized by introducing a strong ferromagnetic coupling limit between these spins in the Hamiltonian. The spin operator is written as

$$
\mathbf{S}_{i}=\sum_{\alpha_{i}=1}^{2 s_{i}} d_{i \alpha_{i}, \sigma}^{\dagger} \frac{\boldsymbol{\sigma}_{\sigma, \sigma^{\prime}}}{2} d_{i \alpha_{i}, \sigma^{\prime}},
$$

with the restriction that each site $i \alpha_{i}$ is singly occupied by $d$-fermions. The effective Hamiltonian in terms of $\mathrm{d}-$ fermions is

$$
\begin{aligned}
& H^{\prime}=\sum_{\langle i j\rangle \in \wedge} t_{i j} c_{i, \sigma}^{\dagger} c_{j, \sigma}+\sum_{i \in \wedge} U\left(n_{i, \uparrow}-\frac{1}{2}\right)\left(n_{i, \downarrow}-\frac{1}{2}\right) \\
& +J \sum_{i, \alpha_{i}}\left(d_{i \alpha_{i}, \sigma}^{\dagger} \frac{\boldsymbol{\sigma}_{\sigma, \sigma^{\prime}}}{2} d_{i \alpha_{i}, \sigma^{\prime}}\right) \cdot\left(c_{i, \sigma}^{\dagger} \frac{\boldsymbol{\sigma}_{\sigma, \sigma^{\prime}}}{2} c_{i, \sigma^{\prime}}\right) \\
& +\sum_{i j \in \wedge, \alpha_{i}, \alpha_{j}} K_{i j}\left(d_{i \alpha_{i}, \sigma}^{\dagger} \frac{\boldsymbol{\sigma}_{\sigma, \sigma^{\prime}}}{2} d_{i \alpha_{i}, \sigma^{\prime}}\right) \cdot\left(d_{j \alpha_{j}, \sigma}^{\dagger} \frac{\boldsymbol{\sigma}_{\sigma, \sigma^{\prime}}}{2} d_{j \alpha_{j}, \sigma^{\prime}}\right) \\
& -\sum_{i \in \wedge} \lambda_{i}\left[\left(\sum_{\alpha_{i}} d_{i \alpha, \sigma}^{\dagger} \frac{\boldsymbol{\sigma}_{\sigma, \sigma^{\prime}}}{2} d_{i \alpha, \sigma^{\prime}}\right)^{2}-s_{i}\left(s_{i}+1\right)\right]
\end{aligned}
$$


where all $\lambda_{i}$ are positive 15 . We shall take all $\lambda_{i} \rightarrow \infty$ at the last step (as a matter of fact our theorem is true for any finite $\lambda_{i}$ ), and this guarantees that any deviation from

$$
\left\langle\Psi\left|\left(\sum_{\alpha_{i}=1}^{2 s_{i}} d_{i \alpha_{i}, \sigma}^{\dagger} \frac{\boldsymbol{\sigma}_{\sigma, \sigma^{\prime}}}{2} d_{i \alpha_{i}, \sigma}\right)^{2}\right| \Psi\right\rangle=s_{i}\left(s_{i}+1\right)
$$

will lead to the divergence of the eigenvalues of energy in Eq. (12). Eq. (13) is an alternative expression of

$$
\mathbf{S}_{i}^{2}|\Psi\rangle=s_{i}\left(s_{i}+1\right)|\Psi\rangle
$$

in the multi-fermion representation. In the limit of $\lambda_{i} \rightarrow+\infty, H^{\prime}$ in Eq. (12) is equivalent to $H$ in Eq. (1).

The definition of the generalised bipartite lattice is very crucial for utilising the reflection positivity technique in the spin space. The generalised bipartite lattice for $H^{\prime}$ is defined as follows: 1). The sublattice $A(B)$ for c-electrons belongs to the generalised sublattice $\mathcal{A}$ $(\mathcal{B}) ; 2)$. The site $i \alpha_{i}$ for $d$-fermions belongs to the generalised sublattice $\mathcal{A}(\mathcal{B})$ if $i$ belongs to the sublattice $A$ $(B)$ when $J_{i}<0$ and to the generalised sublattice $\mathcal{B}(\mathcal{A})$ when $J_{i}>0$. At the same site $i$ all $i \alpha_{i}$ with different $\alpha_{i}$ belong to the same generalised sublattice. Additionally, the sites of the generalised lattice which are connected by $K_{i j}$ belongs to the same sublattice when $K_{i j}<0$ and to two different sublattices when $K_{i j}>0$ according to the condition on the signs of $J_{i}$ and $K_{i j}$.

According to this generalised bipartite lattice, the transformation operator $\mathbf{T}$ for the partial particle-hole transformation is introduced as 11

$$
\mathbf{T}=\prod_{i \in \wedge}\left(c_{i \uparrow}-\epsilon(i) c_{i \uparrow}^{\dagger}\right) \prod_{i \in \wedge_{d}} \prod_{\alpha_{i}=1}^{2 s_{i}}\left(d_{i \alpha_{i} \uparrow}+\frac{J_{i}}{\left|J_{i}\right|} \epsilon(i) d_{i \alpha_{i} \uparrow}^{\dagger}\right) .
$$

Under this transformation, we have

$$
\begin{aligned}
\mathbf{T} c_{i \uparrow} \mathbf{T}^{\dagger} & =(-1)^{N_{t}} \epsilon(i) c_{i \uparrow}^{\dagger} ; \\
\mathbf{T} c_{i \downarrow} \mathbf{T}^{\dagger} & =(-1)^{N_{t}} c_{i \downarrow} ; \\
\mathbf{T} d_{i \alpha_{i} \uparrow} \mathbf{T}^{\dagger} & =(-1)^{N_{t}+1} \frac{J_{i}}{\left|J_{i}\right|} \epsilon(i) d_{i \alpha_{i} \uparrow}^{\dagger} ; \\
\mathbf{T} d_{i \alpha_{i} \downarrow} \mathbf{T}^{\dagger} & =(-1)^{N_{t}} d_{i \alpha_{i} \downarrow},
\end{aligned}
$$

where $N_{t}$ is the total number of the generalised bipartite lattice sites.

The set of basis for $H^{\prime}$ in the case of half filling we choose is $\left\{\mathbf{T}\left|\phi_{\alpha}^{\uparrow}\right\rangle \otimes\left|\phi_{\beta}^{\downarrow}\right\rangle\right\} . \quad\left\{\left|\phi_{\alpha}^{\sigma}\right\rangle\right\}$ is a real, complete and orthonormal set of basis for $N_{0}$ c-electrons and d-fermions with spin $\sigma$, which is expressed as $\phi_{\alpha}^{\sigma}=$ $\prod_{i \in \alpha} c_{i \sigma}^{\dagger} \prod_{i \in \alpha_{i}} d_{i \alpha_{i} \in \alpha}^{\dagger}|0\rangle$ (the order of $c$ and $d$ operators will cause an undetermined phase -1 , which does not affect our final result). The single occupancy of $d$-fermion at site $i \alpha_{i}$ is realized by choosing all $\{\alpha, \beta\}$ such that

$$
n_{i \alpha_{i} \uparrow}\left|\phi_{\alpha}^{\uparrow}\right\rangle \otimes\left|\phi_{\beta}^{\downarrow}\right\rangle=n_{i \alpha_{i \downarrow} \downarrow}\left|\phi_{\alpha}^{\uparrow}\right\rangle \otimes\left|\phi_{\beta}^{\downarrow}\right\rangle
$$

for all $i$ and $i \alpha_{i}$. Each $\left\{\mathbf{T}\left|\phi_{\alpha}^{\uparrow}\right\rangle \otimes\left|\phi_{\beta}^{\downarrow}\right\rangle\right\}$ contains $N_{0}$ celectrons and d-fermions with spin down and $N_{t}-N_{0}$ c-electrons and d-fermions with spin up. The total number of c-electrons and d-fermions in $\left\{\mathbf{T}\left|\phi_{\alpha}^{\uparrow}\right\rangle \otimes\left|\phi_{\beta}^{\downarrow}\right\rangle\right\}$ is $N_{t}$. Consider now the constraint of single occupancy of d-fermions. The number of c-electrons in this basis is $N_{e}=N_{\wedge}$. The z-component of the total spin is

$$
\mathbf{S}_{t o t}^{z} \mathbf{T}\left|\phi_{\alpha}^{\uparrow}\right\rangle \otimes\left|\phi_{\beta}^{\downarrow}\right\rangle=\frac{1}{2}\left(N_{t}-2 N_{0}\right) \mathbf{T}\left|\phi_{\alpha}^{\uparrow}\right\rangle \otimes\left|\phi_{\beta}^{\downarrow}\right\rangle .
$$

Any lowest energy state of $H^{\prime}$ with $S_{t o t}^{z}=\frac{1}{2}\left(N_{t}-2 N_{0}\right)$ can be expanded as

$$
|\Psi(W)\rangle=\sum_{\alpha, \beta} W_{\alpha \beta} \mathbf{T}\left|\phi_{\alpha}^{\uparrow}\right\rangle \otimes\left|\phi_{\beta}^{\downarrow}\right\rangle .
$$

The coefficients $\left\{W_{\alpha \beta}\right\}$ can be regarded as a square matrix and chosen as hermitian since the transformed Hamiltonian $\mathbf{T} H^{\prime} \mathbf{T}^{\dagger}$ possesses spin up-down symmetry.

The proof of the positive definiteness of $\mathrm{W}$ is straightforward, but a little tedious according to the reflection positivity approach in the spin space 6 . The variational energy of $H^{\prime}$ is

$$
\begin{aligned}
E(W) & \equiv\left\langle\Psi(W)\left|H^{\prime}\right| \Psi(W)\right\rangle \\
& =\sum_{\sigma} 2 \operatorname{Tr}\left(W^{2} P\right)-\sum_{i \in \wedge} U_{i} \operatorname{Tr}\left(W V_{i} W V_{i}\right) \\
& -\sum_{i \in \wedge_{d}, \alpha_{i}}\left|J_{i}\right| \operatorname{Tr}\left(W V_{c d i \alpha_{i}} W V_{c d i \alpha_{i}}^{\dagger}\right) \\
& -\sum_{i j \in \wedge_{d}, \alpha_{i}, \alpha_{j}}\left|K_{i j}\right| \operatorname{Tr}\left(W V_{d i \alpha_{i} j \alpha_{j}} W V_{d i \alpha_{i} j \alpha_{j}}^{\dagger}\right) \\
& -\sum_{i \in \wedge_{d}, \alpha_{i}, \alpha_{i}^{\prime}} \lambda_{i} \operatorname{Tr}\left(W V_{d i \alpha \alpha^{\prime}} W V_{d i \alpha \alpha^{\prime}}\right) \\
& +\sum_{i \in \wedge_{d}} \lambda_{i} s_{i}\left(s_{i}+1\right),
\end{aligned}
$$

where

$$
\begin{aligned}
(P)_{\alpha \beta} & =\left\langle\phi_{\alpha}^{\sigma}\right| \sum_{i j \in \wedge} t_{i j} c_{i, \sigma}^{\dagger} c_{j, \sigma} \\
& +\sum_{i \in \wedge_{d}, \alpha_{i}} \frac{J_{i}}{2}\left(n_{i, \sigma}-\frac{1}{2}\right)\left(n_{i \alpha_{i}, \sigma}-\frac{1}{2}\right) \\
& +\sum_{i j \in \wedge_{d}, \alpha_{i}, \alpha_{j}} \frac{K_{i j}}{2}\left(n_{i \alpha_{i}, \sigma}-\frac{1}{2}\right)\left(n_{j \alpha_{j}, \sigma}-\frac{1}{2}\right) \\
& +\sum_{i \in \wedge_{d}, \alpha_{i}, \alpha_{i}^{\prime}} \frac{\lambda_{i}}{2}\left(n_{i \alpha_{i}, \sigma}-\frac{1}{2}\right)\left(n_{i \alpha_{i}^{\prime}, \sigma}-\frac{1}{2}\right)\left|\phi_{\beta}^{\sigma}\right\rangle,
\end{aligned}
$$

and 


$$
\begin{aligned}
\left(V_{i}\right)_{\alpha \beta} & =\left\langle\phi_{\alpha}^{\sigma}\left|\left(n_{i \sigma}-\frac{1}{2}\right)\right| \phi_{\beta}^{\sigma}\right\rangle, \\
\left(V_{c d i \alpha_{i}}\right)_{\alpha \beta} & =\left\langle\phi_{\alpha}^{\sigma}\left|c_{i \sigma} d_{i \sigma}^{\dagger}\right| \phi_{\beta}^{\sigma}\right\rangle, \\
\left(V_{d i \alpha_{i} j \alpha_{j}}\right)_{\alpha \beta} & =\left\langle\phi_{\alpha}^{\sigma}\left|d_{i \alpha_{i}, \sigma} d_{j \alpha_{j} \sigma}^{\dagger}\right| \phi_{\beta}^{\sigma}\right\rangle, \\
\left(V_{d i \alpha_{i} \alpha_{i}^{\prime}}\right)_{\alpha \beta} & =\left\langle\phi_{\alpha}^{\sigma}\left|d_{i \alpha_{i}, \sigma} d_{i \alpha_{i}^{\prime}, \sigma}^{\dagger}\right| \phi_{\beta}^{\sigma}\right\rangle .
\end{aligned}
$$

As $W$ is hermitian and can be decomposed as $W=$ $V^{\dagger} D V$ where $V^{\dagger} V=1$ and $D$ is a diagonal matrix with the diagonal elements $d_{\alpha}$, denoted by $D=\operatorname{diag}\left\{d_{\alpha}\right\}$. Denote $|W|=V^{\dagger}|D| V$ where $|D|=\operatorname{diag}\left\{\left|d_{\alpha}\right|\right\} .|W|$ is at least positive semidefinite as all its eigenvalues are nonnegative. When all $d_{\alpha} \neq 0,|W|$ is positive definite. As

$$
\begin{array}{r}
\operatorname{Tr}\left(W^{2} P\right)=\operatorname{Tr}\left(|W|^{2} P\right) \\
\operatorname{Tr}\left(W X W X^{\dagger}\right) \leq \operatorname{Tr}\left(|W| X|W| X^{\dagger}\right)
\end{array}
$$

and all $U_{i} \geq 0$ we have

$$
E(W) \geq E(|W|) .
$$

If $|\Psi(W)\rangle$ is the lowest energy state, $|\Psi(|W|)\rangle$ must be also the lowest energy state in the variational principle. This indicates that one of the lowest energy states of $H^{\prime}$ if degenerate is at least positive semidefinite. Suppose $|W|$ is positive semi-definite (not positive definite) and there must exist one non-zero vector $V_{0}$ such that $|W| V_{0}=0$. From the Schrödinger equation for $|W|$ and using the variational principle, we have

$$
\begin{aligned}
& |W| N_{c i} V_{0}=0, \quad \text { if } U_{i}>0, \\
& |W| H_{0} V_{0}=0 \\
& |W| V_{\text {cdia }_{i}} V_{0}=0, \quad \text { if } J_{i} \neq 0, \\
& |W| V_{d i \alpha_{i} j \alpha_{j}} V_{0}=0 \text {, if } K_{i j} \neq 0 \text {, } \\
& |W| V_{d i \alpha_{i} \alpha_{i}} V_{0}=0,
\end{aligned}
$$

where

$$
\begin{aligned}
\left(N_{c i}\right)_{\alpha \beta} & =\left\langle\phi_{\alpha}^{\sigma}\left|n_{i \sigma}\right| \phi_{\beta}^{\sigma}\right\rangle, \\
\left(H_{0}\right)_{\alpha \beta} & =\sum_{\langle i j\rangle \in \wedge} t_{i j}\left\langle\phi_{\alpha}^{\sigma}\left|c_{i \sigma}^{\dagger} c_{j \sigma}\right| \phi_{\beta}^{\sigma}\right\rangle .
\end{aligned}
$$

Eq. (36) is obtained from the single occupancy condition of $d$-fermions. When all $U_{i}>0$ and $J_{i} \neq 0$, the generalised lattice is connected through $t_{i j}$ and $J_{i}$. In this case, we can show that for any non-zero vector $V_{a n y}$ we have $|W| V_{a n y}=0$ by successively using Eqs. (32-36). As $|W|$ is an non-zero matrix, it is impossible that all of its eigenvalues are equal to zero. This indicates that $|W|$ is positive definite and non of its eigenvalues are equal to zero. If there are two positive definite states, for instance $W_{1}$ and $W_{2}$, constructing the state $W_{1}+c W_{2}$ ( $c$ is a nonzero constant) and repeating the same procedure above we can show that $W_{1}=W_{2}$ or $W_{1}=-W_{2}$. Thus we conclude that the lowest energy state in each subspace $S_{t o t}^{z}$ is non-degenerate and positive definite. This conclusion holds even if some of $J_{i}=0$ but the generalised lattice is connected through $t_{i j}, J_{i}$ and $K_{i j}$. However we cannot show the non-degeneracy of the lowest energy state in each subspace if the generalised lattice is not connected or not all $U_{i}>0$ except for some special cases. A detailed proof for the case of $s=1 / 2$ is seen in Ref. 8. In principle we have reduced the Kondo model with large spin $\alpha_{i}$ to the model with spin $1 / 2$. The proof for the case of $1 / 2$ can be applied to the case of large spin.

Since the lowest energy state is positive definite on the basis we chogse, a theorem on a positive definite or semidefinite state 9 can be applied to show Theorem (iiiv). The theorem states:

Given a positive semidefinite state $|\Phi\rangle$ on a bipartite lattice with $N_{A}$ and $N_{B}$, then

$1)$. If the state is an eigenstate of the total spin, the eigenvalue $S$ is $\left|N_{A}-N_{B}\right| / 2$ if $\left|S^{z}\right| \leq\left|N_{A}-N_{B}\right| / 2$, and $\left|S^{z}\right|$ otherwise;

2). The transverse spin-spin correlation function (for fermions with spin 1/2) obeys

$$
\left\langle\Phi\left|\mathbf{S}_{i}^{+} \cdot \mathbf{S}_{j}^{-}\right| \Phi\right\rangle=\epsilon(i) \epsilon(j) C_{i j}
$$

where $C_{i j} \geq 0$ ( the equality only holds possibly in a positive semidefinite, not definite state).

In our case, the difference of the total numbers of the two generalised sublattice $\mathcal{A}$ and $\mathcal{B}$ sites is $\sum_{i \in \wedge} \epsilon(i)-$ $\sum_{i \in \wedge_{d}} \frac{J_{i}}{\left|J_{i}\right|} 2 s_{i} \epsilon(i)$. We get Theorem (ii) and (iv) combining the theorem above and the positive definiteness of the lowest energy state. Theorem (iii) is also obtained as we have decomposed the spin $\mathbf{S}_{i}$ into $2 s_{i} 1 / 2$-spins and the whole system is still on a generalised bipartite lattice. For example,

$$
\begin{aligned}
\left\langle\Psi\left|\mathbf{S}_{i}^{+} \cdot \mathbf{S}_{j}^{-}\right| \Psi\right\rangle & =\sum_{\alpha_{i}, \alpha_{j}}\left\langle\Psi\left|d_{i \alpha_{i}, \uparrow}^{\dagger} d_{i \alpha_{i}, \downarrow} d_{i \alpha_{i}, \downarrow}^{\dagger} d_{i \alpha_{i}, \uparrow}\right| \Psi\right\rangle \\
& =\epsilon(i) \epsilon(j) F_{i j}
\end{aligned}
$$

where

$$
F_{i j}=\sum_{\alpha_{i}, \alpha_{j}} \operatorname{Tr}\left(W^{\dagger} V_{d i \alpha_{i} j \alpha_{j}} W\left(V_{d i \alpha_{i} j \alpha_{j}}\right)^{\dagger}\right) .
$$

$F_{i j}>0$ when the state is positive definite, and $\geq 0$ when the state is positive semidefinite.

In summary, we have provided several theorems on the ground state properties of the Kondo model at half-filling and in the case with large spin. The uniqueness and the total spin in the ground state are found. Furthermore we have also investigated the spin-spin correlation in the system. The co-existence of both antiferromagnetic and ferromagnetic long-range correlations is proved, which is similar to the case of spin $1 / 2$. Hopefully, these exact results are useful for understanding this highly correlated electron-impurity lattice.

I would like to thank Prof. P. Fulde for conversations. I also wish to thank Dr. D. F. Wang for fruitful discussions and for his encouragement. This work was supported by the Alexander von Humboldt foundation of Germany. 
${ }^{1}$ For a review, please see P. Fulde, J. Keller, and G. Zwicknagl, In Solid State Physics, Vol. 41, ed. by H. Ehrenreich and D. Turnbull, 1 (Academic, San Diego 1988), P. A. Lee, T. M. Rice, J. W. Serene, L. J. Sham and J. W. Willkins, Comments Condens. Matt Phys. 12, 99 (1986), and P. Coleman, Phys. Rev. B 28, 5255 (1983). References therein.

${ }^{2}$ P. Coleman, E. Mirada and A. Tsvelik, Phys. Rev. B 49, 7252 (1989).

${ }^{3}$ I. Affleck and A. Ludwig, Nucl. Phys. B 360, 641 (1991), B 352, 849 (1991).

${ }^{4}$ E. H. Lieb, Phys. Rev. Lett. 62, 1201; 1927(E) (1989).

${ }^{5}$ K. Ueda, H. Tsunetsugu, and M. Sigrist, Phys. Rev. Lett. 68, 1030 (1992).

${ }^{6}$ S. Q. Shen, Z. M. Qiu, and G. S. Tian, Phys. Rev. Lett. 72, 1280 (1994).

${ }^{7}$ T. Yanagisawa and Y. Shimoi, Phys. Rev. Lett. 74, 4939 (1995).

${ }^{8}$ S. Q. Shen, Phys. Rev. B 53, 14252 (1996).
${ }^{9}$ S. Q. Shen, Phys. Rev. B 54, 4397 (1996).

10 J. Inoue and S. Maekawa, Phys. Rev. Lett. 74, 3407 (1995).

${ }^{11}$ F. C. Pu and S. Q. Shen, Phys. Rev. B 50, 16086 (1994).

12 E. H. Lieb and D. C. Mattis, J. Math. Phys. 3, 749 (1962).

13 S. R. White and I. Affleck, Phys. Rev. B 54, 9862 (1996).

${ }^{14}$ Usually we say a square matrix or an operator is positive definite or semidefinite when all its eigenvalues are positive or non-negative. In theory of reflection positivity, the state is expressed as a square matrix on a chosen basis. When we say a state is positive definite or semidefinite, it means that the matrix standing for the state is positive definite or semidefinite.

${ }^{15}$ We can also regard the last term in Eq. (12) as Lagrange multipliers $\lambda_{i}$. In this case, they are variable, not constant. The resulting variational conditions are the same as those in Eq. (13). As $s_{i}\left(s_{i}+1\right)$ is the maximum of the eigenvalues of $\mathbf{S}_{i}^{2}$, the resulting conditions are equivalent to those in Eq. (14). 